\title{
Simulation of an Electromechanical Spin Motor System of a Control Moment Gyroscope
}

\author{
Ravi Inampudi* and John Gordeuk ${ }^{\dagger}$
}

\begin{abstract}
A two-phase brushless DC motor (BDCM) with pulse-width modulated (PWM) voltage drive is simulated to control the flywheel speed of a control moment gyroscope (CMG). An overview of a double-gimballed control moment gyroscope (DGCMG) assembly is presented along with the CMG torque effects on the spacecraft. The operating principles of a two-phase brushless DC motor are presented and the system's electro-mechanical equations of motion are developed for the root-mean-square (RMS) currents and wheel speed. It is shown that the system is an extremely "stiff" set of first-order equations for which an implicit Euler integrator is required for a stable solution. An adaptive proportional voltage controller is presented which adjusts the PWM voltages depending on several control modes for speed, current, and torque. The simulation results illustrate the interaction between the electrical system and the load dynamics and how these influence the overall performance of the system. As will be shown, the CMG spin motor model can directly provide electrical power use and thermal power output to spacecraft subsystems for effective (average) calculations of CMG power consumption.
\end{abstract}

\section{Introduction}

Training systems for the 21st Century (TS21) at JSC will provide a simulation-based training for crew members, instructors, and flight controllers on the operation of FOD (Flight Operations Directorate) supported spacecraft including the International Space Station (ISS), Robotics, ISS Visiting Vehicles and other future NASA owned crew transport like MPCV (Multi-Purpose Crew Vehicle). TS21 products include the simulation architecture and math models for the space environment, robotics, and vehicle subsystems. ${ }^{1}$ The 6-dof state (position and attitude) of the space station is one of the most fundamental components for all ISS operations. The ISS Attitude Determination and Control Officer (ADCO) has overall responsibility for the integration of all Guidance, Navigation and Control (GNC), including propulsive and CMG attitude control. The ADCO works in partnership with Russian controllers to manage the station's orientation, controlled by the on-board Motion Control Systems. They also plan and calculate future orientations and maneuvers for the station.

The CMG cluster mounted on the ISS is a set of 4 spinning wheels gimballed to provide long-term attitude control as well as to store momentum. A CMG is a momentum exchange device consisting of a mechanical momentum wheel assembly with supporting gimbals (inner/outer) and electronic equipment. The spin-wheel assembly consists of a flywheel, a spin-motor, and a hall resolver to sense rotor speed.

\footnotetext{
${ }^{*}$ Lead Software Engineer, Training systems for the 21st Century (TS21), Lockheed Martin, Houston, Texas, 77058, AIAA Member

${ }^{\dagger}$ Senior Engineer, Training systems for the 21st Century (TS21), GHG Corporation, Webster, Texas, 77598. 
Gimbal motion control allows the CMG cluster to manage the angular momentum and to precisely position and/or point the ISS. For attitude control, the gimbals respond to angular rate commands sent from the GNC flight software (FSW) resulting in reaction torque on the spacecraft structure. The electrical assembly (EA) provides control and monitoring capabilities of the IG, OG and wheel spin functions of the mechanical assembly. The EA also communicates with the GNC FSW through commands and telemetry. For instance, the EA receives and executes commands from the GNC FSW and relays, feedback and sensor information, including Built-In-Test (BIT) data, temperatures, motor currents, rotor unbalances, and rotational speeds. ${ }^{2}$

The main component within the inner gimbal assembly of a CMG is the flywheel which rotates at a nominal speed of 6600 RPM, supplying an angular momentum of about $3500 \mathrm{ft}-\mathrm{lb}$-sec. Gimbal motion results in torque reaction to the CMG mounting ring and then to the spacecraft. A special wheel braking mode allows power generated by the spin motor to be injected into select areas of the power bus internal to the EA. The power supply proper (DC to DC converter), provides power mainly for the spin motor PWM circuit card and the spin motor timing and control circuit card. The spin motor PWM circuit card provides excitation to the two-phase brushless DC motor. This motor provides drive torque to the flywheel, which will reach and maintain a nominal speed of $6600 \mathrm{rpm}$. The spin motor timing circuit uses the hall resolver sine output to control the wheel speed through the phase/frequency detector in the speed control loop. The hall resolver sine and cosine outputs are used for commutation, digital wheel speed determination, and fault isolation. $^{2}$

Reference 2 presents a thorough treatment of CMG mechanical assembly, Reference 4 discusses in detail the CMG dynamics and control and Reference3 specifically covers the dynamics and control of a double gimbaled variable speed CMG. Simulation of flywheel electrical system for aerospace applications is presented in Reference 5. A tutorial style overview of the basic concepts underlying the hardware design and simulation development of electromechanical motion control systems and spacecraft applications is presented in Reference 6. This reference presents various electromechanical motion devices, torque expressions, mechanical systems and their electrical analogs, drive electronics and topologies and control techniques.

This paper simulates a two-phase brushless DC motor with PWM voltage drive to control the flywheel speed of a CMG. Incrementally, the basic working principles of a single-phase, followed by a two-phase brushless DC motor and then the CMG spin motor electromechanics are presented. To simulate a two-phase brushless DC motor, the spin motor system's electro-mechanical equations of motion are developed for the root-mean-square (RMS) currents and wheel speed. A stable solution method is discussed to solve a system of first-order equations for the wheel speed and the sine and cosine currents. A proportional voltage controller is presented which adjusts the PWM voltages depending on several modes to control wheel speed, drive currents and desired torque. Also, the CMG spin motor model can directly provide electrical power use and thermal power output to other ISS spacecraft subsystems. Finally, the simulation results and how these influence the overall performance of the system are discussed.

\section{A. Single-Phase PWM Brushless DC Motor}

\section{PWM Brushless DC Spin Motor}

In order to understand the CMG spin motor dynamics and performance, the interaction between drive electronics, motor and mechanical load must be represented in sufficient detail. As a prelude, this section will focus on the basic mechanics of a single-phase PWM brushless DC motor.

\section{Brushed vs Brushless DC Motors}

A brushed DC motor (BDC) has a stator and is the stationary outside part of a motor which provides a permanent magnetic field and a commutator is attached to the internal armature shaft that keeps reversing the current flow direction to ensure that the motor continuous to turn in the same direction. Just as the rotor reaches alignment, the brushes move across the commutator contacts and energize the next winding. 
More loops are added to the rotor to increase the magnetic field strength. The brushes of a DC motor have several limitations; brush life, brush residue (debris), maximum speed, and sparks and electrical noise. A brushless DC motor (BLDC) solves these problems; this brushless motor topology is created by taking a brushed DC motor and flipping it inside out. So, in a BLDC motor, the brushes are eliminated by moving the outer permanent magnets to the rotor. Now the rotor is a permanent magnet with shaft coming out and bearings on either side of it. And the coils are wrapped around the outside of the stator. Then, how is the motor driven? The trick is to control the current flow to the field coils on the outside with the help of control drive electronics. The control electronics replace the function of a commutator and energize the proper winding at the right time. Therefore, the idea remains the same; some how the magnetic polarity of the rotor coils needs to be changed; in a BLDC motor, instead of mechanical commutators, electronics continuously change the phase of the stator coils to keep the motor rotating. In this condition, when one coil is energized the opposite poles of rotor and stator are attracted to each other. As the rotor nears one coil, the second coil is energized. As the rotor nears the second coil, the third coil is energized. This process is repeated and the rotor continues to rotate. A brushless motor design with coils on the outside and the permanent magnet inside has two advantages: first, more windings for the same diameter of the motor means more current can pass through and hence more magnetic field, so more powerful for the same size. Second, is in terms of cooling. A brushed DC motor gets hot which limits on how much current is put through them and that limits on what can done with it. If the coils are on the outside, heat sinks can be put outside of the motor to get rid of that heat which means increased power density. Also, as brushless motors lack this brush-commutator interface, they exhibit lower acoustic noise.

\section{Feedback Control Using PWM Drive}

How do you control torque on a DC motor? How and when are the stator coils energized to obtain a smooth continuous rotation of the rotor? The parameter that is related to torque is the current. Figure 1 shows a typical negative feedback control system for a single phase DC motor. In a DC motor, commutation keeps the rotor and stator fields properly aligned. Such a field oriented control is performed in a brushed DC motor using a mechanical commutator and brushes whereas in a brushless DC motor it is performed electronically. For both kinds of DC motors, the main ideas of the feedback control are summarized as follows:

1. Measure the current that is already flowing in the motor. Sample the current using either a shunt resistor or a Hall effect sensor.

2. Compare the measured speed (current) with the desired speed (current) and generate a voltage error signal.

3. Amplify the error signal to generate a correction voltage with some type of control algorithm; for example, using a proportional controller.

4. Modulate the correction voltage on the motor terminals; for example, using pulse width modulation (PWM) technique.

These four steps are discrete steps in an interrupt service routine (ISR) and the ISR is repeatedly interrupted at a certain frequency. Over a period, the measured current will converge on to the desired current value. Also, the motor's current is controlled through the motor's voltage. Therefore, voltage is the control variable and increasing the voltage increases the current to the motor and vice versa. A Hall affect sensor tells the controller the motor's position to switch the current to the coils in the right order in order to keep the motor running in the same direction. As shown in Figure 2, a pulse-width modulation(PWM) is a popular method of controlling the motor, which converts an analog-input voltage into a variable-duty-cycle drive signal. Beginning at zero duty cycle (OFF all the time), the duty cycle advances as the motor begins to rotate, until it's running at the speed and/or torque required by the application. 


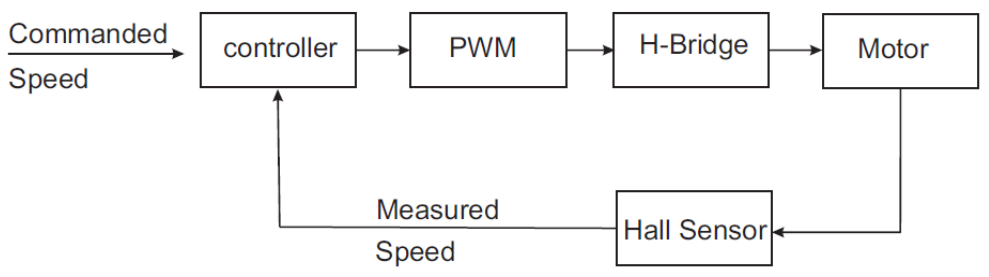

Figure 1: Motor Control Loop With PWM

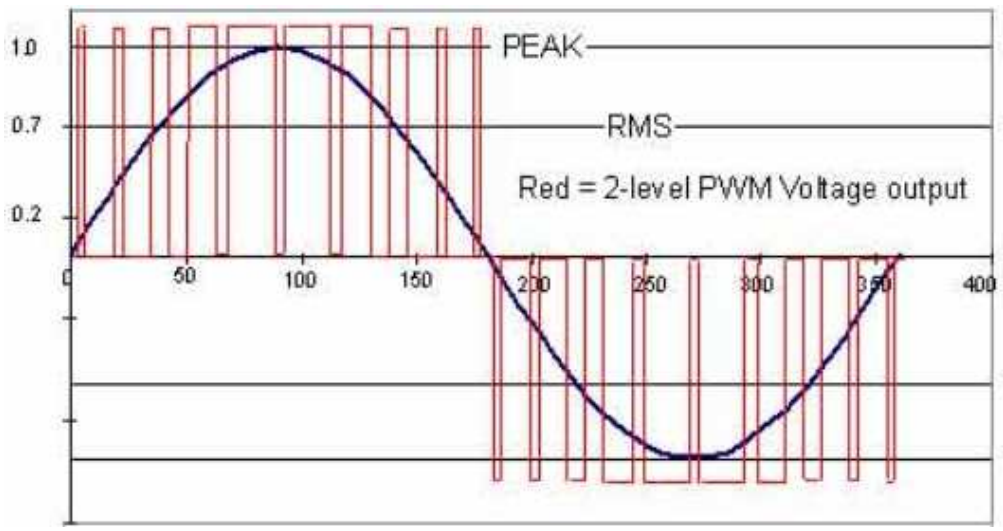

Figure 2: PWM Pseudo Sine Wave

\section{A Single-Phase DC Motor Circuit}

Motors do useful work by creating torque exploiting the relationships between electrical current and magnetic fields. A DC motor is controlled by applying a voltage to the motor terminals and the torque is generated as a result of current flowing in the rotor coils in the presence of a permanent magnetic field (Lorentz force). On the other hand, as the rotor's coil moves through an external magnetic field, an electromotive force (back EMF) generates an opposing voltage thus reducing the voltage drop across the motor's terminals (Faraday's Law). The faster the motor spins, the more the back-EMF is generated. Therefore the back-EMF $V_{\text {bemf }}$ is related to the rotational speed $\omega$ as

$$
V_{\text {bemf }}=K_{\mathrm{e}} \omega
$$

where $V_{\text {bemf }}$ is in Volts, $\omega$ is in rad/s and the speed constant $K_{\mathrm{e}}$ is in $\mathrm{V} / \mathrm{rad} / \mathrm{s}$. The value of $K_{\mathrm{e}}$ is determined from the motor properties such as number of windings in the coil, length of the coil, radius of the winding and magnetic flux density of the permanent magnet. Consequently, the electrical power input, $P_{e}$, to the motor is given as

$$
P_{\mathrm{e}}=V_{\mathrm{bemf}} i
$$

An expression for mechanical power output of the motor, $P_{m}$, is derived from the definition of magnetic force on electric current in a magnetic field. In a DC motor, since the direction of the current $i$ in the wire of length $l$ is perpendicular to the magnetic field $B$, then the magnetic force $F$ is

$$
F=i l B
$$

Assuming a rectangular flat coil, an expression for torque is derived as

$$
\begin{aligned}
T_{\mathrm{m}} & =(\text { number of turns })(\text { Force/turn })(\text { radius of the coil }) \\
& =N(i l B) R \\
& =K_{\mathrm{t}} i
\end{aligned}
$$


where $K_{\mathrm{t}}=2 \mathrm{NlBR}$ is defined as a motor constant $(\mathrm{Nm} / \mathrm{A})$ and is unique to each motor. Consequently, the mechanical power output $P_{m}$ is given as

$$
P_{m}=T_{\mathrm{m}} \omega
$$

Ignoring the frictional losses in the motor, if $T_{\mathrm{m}}$ is the usable torque output generated by the motor then the electrical power input, $P_{e}$, will equal to the mechanical power output, $P_{m}$, which leads to

$$
V_{\text {bemf }} i=T_{\mathrm{m}} \omega
$$

which further yields $K_{\mathrm{e}}=K_{\mathrm{t}}$. It is useful to know that the two constants are equal to each other only in SI units when $K_{\mathrm{t}}$ is given in $\mathrm{Nm} / \mathrm{A}$ and $K_{\mathrm{e}}$ is in $\mathrm{V} / \mathrm{rad} / \mathrm{s}$.

Figure (3) shows an electrical model of a DC motor and a direct application of the Kirchhoff's voltage law to this armature circuit yields

$$
V=i R_{\mathrm{m}}+L_{\mathrm{m}} \frac{\mathrm{d} i}{\mathrm{~d} t}+V_{\mathrm{bemf}}(t)
$$

where $L_{\mathrm{m}}$ is the inductance in $\mathrm{H}$ and $R_{\mathrm{m}}$ is the resistance in Ohms.

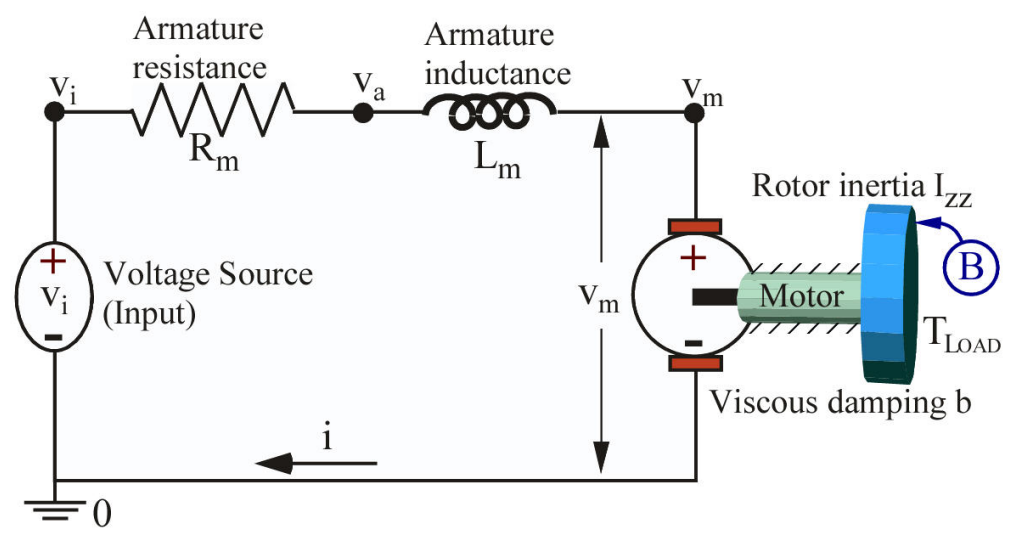

Figure 3: DC Motor Model

Next, assuming that the motor is not connected to any mechanical load and ignoring the friction and core losses of the motor, direct application of Euler's torque equation to the armature circuit yields the mechanical model

$$
J_{\mathrm{m}} \frac{\mathrm{d} \omega_{m}(t)}{\mathrm{d} t}=T_{\mathrm{m}}
$$

Therefore, Eqs. (7) and (8) can be expressed as a set of two first-order differential equations

$$
\begin{aligned}
\frac{\mathrm{d} i}{\mathrm{~d} t} & =\frac{1}{L_{\mathrm{m}}}\left(V(t)-i(t) R_{\mathrm{m}}-\omega(t) K_{\mathrm{e}}\right) \\
\frac{\mathrm{d} \omega}{\mathrm{d} t} & =\frac{K_{\mathrm{t}}}{J_{\mathrm{m}}} i(t)
\end{aligned}
$$

where $R_{m}$ is the stator resistance and $L_{\mathrm{m}}$ is the stator inductance for the winding pair. Voltage $V(t)$ is the control variable and once a voltage is applied to the motor, the rotor begins turning and generates a back EMF. If we assume that the motor is driven by a steady-state voltage $V_{0}$, the closed form solutions of Eqs. (9) for the current $i(t)$ and speed $\omega(t)$ are

$$
\begin{aligned}
i(t) & =\frac{\left(V_{0}-V_{\text {bemf }}\right)}{R_{\mathrm{m}}}\left(1-e^{-\frac{t}{\tau_{e}}}\right) \\
\omega(t) & =\frac{\left(V_{0}-V_{\text {bemf }}\right)}{K_{\mathrm{t}}} \frac{\tau_{e}}{\left(\tau_{m}-\tau_{e}\right)} e^{-\frac{t}{\tau_{e}}}
\end{aligned}
$$


where $\tau_{e}=L_{\mathrm{m}} / R_{\mathrm{m}}$ and $\tau_{m}=J_{\mathrm{m}} / B_{\mathrm{m}}$ are the electrical and mechanical time constants of Eqns. (10). Also, the solutions are obtained with the inherent assumption that $\tau_{e}<<\tau_{m}$. Figure 4 (left side), shows the current when the switch is closed, is the familiar waveform characterizing the current in any L-R circuit, with its rise time governed by the time constant $\tau_{e}$. Also, the maximum current is nearly equal to $\frac{V_{0}}{R_{\mathrm{m}}}$ and is obtained in a very short time after the switch is closed. Now, Figure 4, (right side) shows the current when the steady-state excitation voltage is replaced with a PWM source. The current rises until the first ON pulse ends. At the end of the pulse, when the applied voltage abruptly falls to zero (OFF), the current begins to decay toward zero. However, the next ON pulse will again drive the current upwards, and so forth; the current to the motor continues to rise. As the motor accelerates, the current waveform exhibits a ripple which is saw-tooth profile with increasing amplitude. Because torque is directly proportional to current, this ripple develops a corresponding torque that not only drives but also accelerates the motor. Applied voltage, switching frequency, and the PWM duty cycle are three crucial parameters that determines a motor's speed, acceleration and its torque generation.
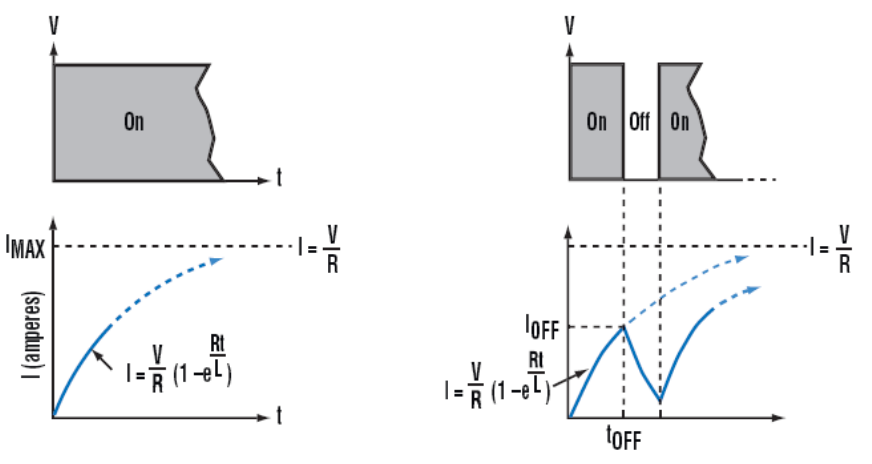

\section{Figure 4: Steady-state Excitation (left) vs PWM Excitation (right)}

\section{CMG Spin Motor Electromechanics}

There are four CMGs on board the ISS shown in Figure 5(a) and each CMG has a spin motor which uses a two-Phase PWM brushless DC motor. Each spin motor consists of a permanent-magnet rotor, a twophase-wound stator, and a set of magnetic Hall devices to sense rotor position. The 8-pole brushless DC motor is pulse-width modulated (PWM) at $50 \mathrm{kHz}$ so as to provide a smooth pseudo-sine wave current to each of the two phases. Figure 5(b) shows 8-poles in 4 pole-pairs which push-pull on the 8-poles on the rotor. The two separately excited circuits drive the shaft in quadrature applying their push-pull torques $90^{\circ}$ apart thus defining the sine and cosine phase drives. Each circuit is driven by its own H-Bridge which is fed a DC voltage from the ISS power bus (nominally 120 volts) so that if one motor fails the other keeps providing the needed torque. The sine and cosine coils each apply a torque waveform similar to a rectified sinusoid with $90^{\circ}$ related phase. The sum of the two waveforms provides a smoother average torque to the rotor.

\section{A. Electrical Model}

The differential equation for $\mathrm{d} i / \mathrm{d} t$ in Eq. 9a is an explicit function of time and instead of solving for an exact time solution, the individual sine/cosine waves of currents and voltages or the back EMF and phases between them are found using simple effective relationships among the RMS currents and voltages. A simplified model averages the sine wave fluctuations and gives out effective RMS currents and voltages and in a way it simulates a direct DC model. So, an RMS value is created by taking the peak value of the sine wave and scaling its amplitude by $\sqrt{2}$ which eliminated the need to solve for the phase difference as the phase falls away with an RMS computation. The aim is to generate constant RMS currents and voltages 


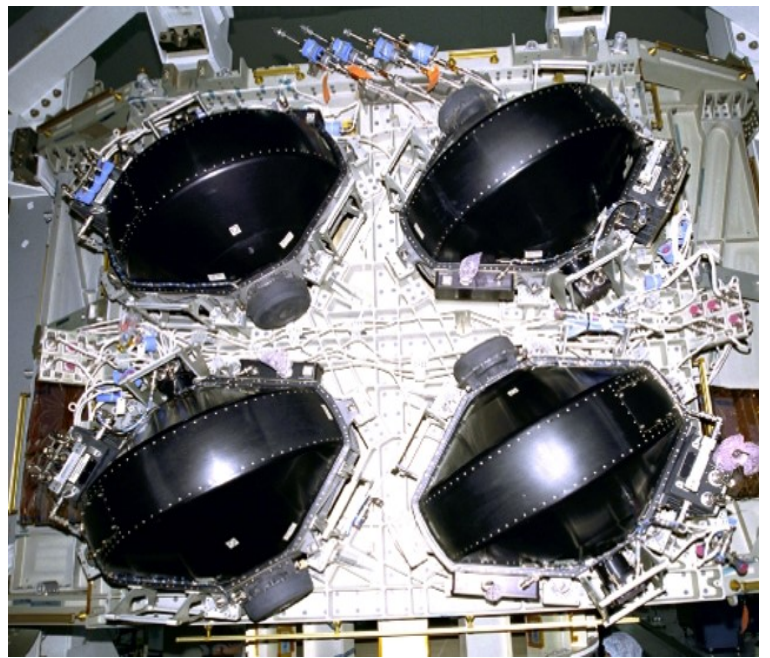

a) 4 CMGs

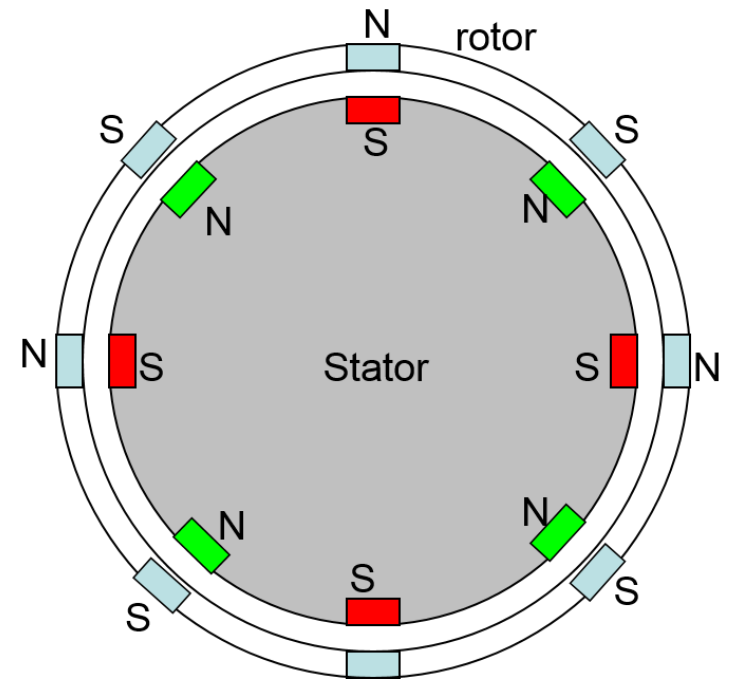

b) Spin Motor

Figure 5: ISS CMGs

such that the motor spins at a constant RPM from which we derive the parameters such as resistance of the motor and the power it uses. As a consequence, the power and resistance becomes the load on the space stations. Using sine waves with amplitudes relative to the peak value of each quantity and rearranging and rewriting Ohm's law gives

$$
L \frac{\mathrm{d} i_{s}}{\mathrm{~d} t}=V \sin \left(\omega_{e} t-\theta\right)-R i \sin \left(\omega_{e} t-\phi\right)-K_{\mathrm{e}} \omega \sin \left(\omega_{e} t\right)
$$

Since the back EMF peaks when the magnets/coils line up we will call this zero phase. The motor controller modulates $V, \omega_{e}$ and $\theta$ to create a smooth push-pull action on the rotor but the current will be at some arbitrary phase $\phi$. It seems natural that the $V$ would lead the $E$ (advanced timing) and the $i$ would lag behind $E$. The equation 11 gives the effective averages of these terms. To simulate a simple effective model for currents and voltages in the motor, standard root mean square (RMS) values of $V$ and $i$ are used which is typical in AC circuit analysis.

$$
L \frac{\mathrm{d} i}{\mathrm{~d} t}{ }_{\mathrm{rms}}=\frac{V_{\text {peak }}}{\sqrt{2}}-R \frac{L_{\text {peak }}}{\sqrt{2}}-\omega \frac{K_{\mathrm{e}-\mathrm{peak}}}{\sqrt{2}}=V_{\mathrm{rms}}-R i_{\mathrm{rms}}-\omega \frac{K_{\mathrm{e}}}{\sqrt{2}}
$$

The effective RMS values are not affected by frequency or phase differences. Rewriting another equation for the cosine phase gives

$$
L \frac{\mathrm{d} i_{c}}{\mathrm{~d} t}=V_{c} \cos \left(\omega_{e} t-\theta\right)-R i_{c} \cos \left(\omega_{e} t-\phi\right)-K_{e} \omega \cos \left(\omega_{e} t\right)
$$

As the RMS equation for cosine is similar to the sine current, it yields a set of two equations

$$
\begin{aligned}
L \frac{\mathrm{d} i_{s}}{\mathrm{~d} t} & =V_{s}-R i_{s}-\omega \frac{K_{\mathrm{e}}}{\sqrt{2}} \\
L \frac{\mathrm{d} i_{c}}{\mathrm{~d} t} & =V_{c}-R i_{c}-\omega \frac{K_{\mathrm{e}}}{\sqrt{2}}
\end{aligned}
$$

Most of the time $V_{s}$ will equal $V_{c}$ unless a drive is failed in some manner. Each drive applies its own separate torque to the rotor $90^{\circ}$ out-of-phase with each other. But the torques must use the push-pull action to drive the rotor in the same direction to speed up or slow down. Therefore the torque is defined with a constant, $T=K_{\mathrm{t}} i$ where $K_{\mathrm{t}}$ is in $\frac{\mathrm{Nm}}{\text { Amps-peak }}$. Even though $i$ is a sine wave that goes positive-negative (pushpull), the torque must be driven always positive to spin up (push-push or pull-pull). 


\section{B. Mechanical Model}

The torque is a fluctuating function in time, but an effective average value of the torque is desired. Here an RMS value is not used but the average value over a cycle that is normally used in mechanics. Summing up the two drive torque gives

$$
T_{\text {total }}=K_{\mathrm{t}} i_{c}\left|\cos \left(\omega_{e} t-\theta\right)\right|+K_{\mathrm{t}} i_{s}\left|\sin \left(\omega_{e} t-\theta\right)\right|
$$

Using the definition for the average of a function

$$
T_{\text {ave }}=\frac{1}{P} \int_{0}^{p} K_{\mathrm{t}} i_{c}|\cos | d t+\frac{1}{P} \int_{0}^{p} K_{\mathrm{t}} i_{s}|\sin | d t
$$

where $P$ is the period. A little research reveals that

$$
\frac{1}{P} \int_{0}^{p}|\sin | d t=\frac{1}{P} \int_{0}^{p}|\cos | d t=\frac{2}{\pi}
$$

As $K_{\mathrm{t}}$ is defined with peak-amps we have

$$
T_{\text {ave }}=K_{\mathrm{t}} \frac{2}{\pi}\left(i_{c}+i_{s}\right)_{\text {peak }}
$$

It is realized that $i_{c}$ and $i_{s}$ are the peak values of our sine and cosine currents. But from AC circuit analysis, $i_{\text {peak }}=\sqrt{2} i_{\text {rms }}$. So using the RMS currents for average torque

$$
T_{\mathrm{ave}}=K_{\mathrm{t}} \frac{2 \sqrt{2}}{\pi}\left(i_{c}+i_{s}\right)_{\mathrm{rms}}
$$

Plugging in this torque into the dynamics equation 9 (with viscous drag) gives

$$
J_{\mathrm{m}} \frac{\mathrm{d} \omega}{\mathrm{d} t}=\frac{2 \sqrt{2}}{\pi} K_{\mathrm{t}}\left(i_{c}+i_{s}\right)_{\mathrm{rms}}-B_{\mathrm{m}} \omega
$$

where $J_{\mathrm{m}}$ is the wheel inertia and $B_{\mathrm{m}}$ is the viscous drag constant.

\section{Solution Method}

The dynamic variables that are solved for are the sine and cosine currents $\left(I_{s}\right.$ and $\left.I_{c}\right)$ as well as motor speed $\omega$. Therefore, the state vector $\boldsymbol{x}$ is

$$
\boldsymbol{x}=\left(I_{c}, I_{s}, \omega\right)
$$

And a set of first-order differential equations for currents and speed where the motor constants and state variables have been converted to their effective RMS values is

$$
\begin{aligned}
\frac{\mathrm{d} I_{c}}{\mathrm{~d} t} & =\frac{1}{L}\left(V_{c}-I_{c} R-\omega K_{\mathrm{e}}\right) \\
\frac{\mathrm{d} I_{s}}{\mathrm{~d} t} & =\frac{1}{L}\left(V_{s}-I_{s} R-\omega K_{\mathrm{e}}\right) \\
\frac{\mathrm{d} \omega}{\mathrm{d} t} & =\frac{1}{J_{\mathrm{m}}}\left(I_{c} K_{\mathrm{t}}+I_{s} K_{\mathrm{t}}-\omega B\right)
\end{aligned}
$$


For a numerical solution, the derivatives in Eq.(22) are approximated as first-order difference equations and rewritten them as

$$
\begin{aligned}
I_{c 1} & =I_{c 0}+\frac{\Delta t}{L}\left(V_{c}-I_{c 1} R-\omega_{1} K_{\mathrm{e}}\right) \\
I_{s 1} & =I_{s 0}+\frac{\Delta t}{L}\left(V_{s}-I_{s 1} R-\omega_{1} K_{\mathrm{e}}\right) \\
\omega_{1} & =\omega_{0}+\frac{\Delta t}{J_{\mathrm{m}}}\left(I_{c 1} K_{\mathrm{t}}+I_{s 1} K_{\mathrm{t}}-\omega_{1} B\right)
\end{aligned}
$$

where the subscripts 1 and 0 denote new and previous values. Applying naively a simple Euler method to the above equations and assuming all quantities on the right side are previous values, quickly yields unstable currents and speeds and eventually blowing up to very large values. Here is a case of a big motor driving the heavy wheel with huge inertia thus creating an ultra-stiff set of differential equations. This is illustrated by comparing the time constants in the electrical and mechanical equations. Using the parameters from Table 1, the electrical time constant $\tau_{e} \approx 0.6 \mathrm{~ms}$ is roughly 8 orders of magnitude separating the mechanical inertia time constant $\tau_{m} \approx 139000 \mathrm{~s}$. The electrical system responds similar to a woofer in a home speaker system whereas the mechanical system would take days to spin down on its own. Consequently, to solve for the three states, an implicit Euler integrator is used for two coupled equations. Therefore, the solution to this problem is of the form

$$
\begin{aligned}
& x_{1}=x_{0}+\Delta t f\left(x_{1}, y_{1}\right) \\
& y_{1}=y_{0}+\Delta t g\left(x_{1}, y_{1}\right)
\end{aligned}
$$

Where the derivative functions contain the new values of the states. Writing the system of equations Eq.(23) in matrix form gives

$$
\left[\begin{array}{lll}
a & 0 & b \\
0 & a & b \\
c & c & d
\end{array}\right]\left[\begin{array}{c}
I_{c 1} \\
I_{s 1} \\
\omega_{1}
\end{array}\right]=\left[\begin{array}{c}
I_{c 0}+V_{c} \frac{\Delta t}{L} \\
I_{s 0}+V_{s} \frac{\Delta t}{L} \\
\omega_{0}
\end{array}\right]
$$

Where $a=1+\frac{R}{L} \Delta t, b=\frac{K_{\mathrm{e}}}{L} \Delta t, c=\frac{-K_{\mathrm{t}}}{J_{\mathrm{m}}} \Delta t$ and $d=1+\frac{B}{J_{\mathrm{m}}} \Delta t$. Solve for the new states by inverting the above matrix:

$$
\left[\begin{array}{c}
I_{c 1} \\
I_{s 1} \\
\omega_{1}
\end{array}\right]=\left[\begin{array}{ccc}
a & 0 & b \\
0 & a & b \\
c & c & d
\end{array}\right]^{-1}\left[\begin{array}{c}
I_{c 0}+V_{c} \frac{\Delta t}{L} \\
I_{s 0}+V_{s} \frac{\Delta t}{L} \\
\omega_{0}
\end{array}\right]=g\left[\begin{array}{ccc}
f & e & -b \\
e & f & -b \\
-c & -c & a
\end{array}\right]\left[\begin{array}{c}
I_{c 0}+V_{c} \frac{\Delta t}{L} \\
I_{s 0}+V_{s} \frac{\Delta t}{L} \\
\omega_{0}
\end{array}\right]
$$

where $e=\frac{b c}{a}, f=d-e, g=\frac{1}{a d-2 b c}=\frac{1}{a f-b c}$. An efficient 2-step algorithm for calculating new states is:

$$
\begin{aligned}
I_{c 0} & =I_{c}+V_{c} \frac{\Delta t}{L} \\
I_{s 0} & =I_{s}+V_{s} \frac{\Delta t}{L} \\
\omega_{0} & =\omega
\end{aligned}
$$

and

$$
\begin{aligned}
I_{c} & =g\left(f I_{c 0}+e I_{s 0}-b \omega_{0}\right) \\
I_{s} & =g\left(e I_{c 0}+f I_{s 0}-b \omega_{0}\right) \\
\omega & =g\left(-c I_{c 0}-c I_{s 0}+a \omega_{0}\right) \\
\Delta \omega & =\omega-\omega_{0}
\end{aligned}
$$


The external driving voltages are $V_{c}$ and $V_{s}$ which are also the control variables. The change in wheel speed $\Delta \omega$ is a useful quantity.

\section{Power Calculations}

The spin motor is activated by an electrical power supply which feeds the PWM circuit driving the motor. The power used is converted to mechanical spin of the wheel, to resistance losses in the windings as heat, and friction in the bearings. With the currents estimated earlier, it is straightforward to calculate the power use $(I V)$. A good estimate of the total power used is derived by converting the state equations 22 into power equations. Multiplying each electrical equation by its corresponding current yields

$$
\begin{aligned}
& I_{\mathrm{s}} L \frac{\mathrm{d} I_{\mathrm{s}}}{\mathrm{d} t}=V_{s} I_{\mathrm{s}}-R I_{s}^{2}-\omega K_{\mathrm{e}} I_{\mathrm{s}} \\
& I_{\mathrm{c}} L \frac{\mathrm{d} I_{\mathrm{c}}}{\mathrm{d} t}=V_{c} I_{\mathrm{c}}-R I_{c}^{2}-\omega K_{\mathrm{e}} I_{\mathrm{c}}
\end{aligned}
$$

Then multiplying the mechanical equation by the speed $\omega$

$$
\omega J_{\mathrm{m}} \frac{\mathrm{d} \omega}{\mathrm{d} t}=\omega K_{\mathrm{t}}\left(I_{c}+I_{s}\right)_{\mathrm{rms}}-B_{\mathrm{m}} \omega^{2}
$$

As shown in section 3., the motor constants $K_{\mathrm{t}}$ and $K_{\mathrm{e}}$ are equivalent when expressed in the same units. Thus, $\omega K_{\mathrm{t}} I_{s, c}=\omega K_{\mathrm{e}} I_{s, c}$. Summing the two electrical Eqs (29) and substituting in the motor constant terms from the mechanical equation 30 and assuming that the currents are slowly changing $\left(\frac{\mathrm{d} I_{\mathrm{s}, \mathrm{c}}}{\mathrm{d} t} \approx 0\right)$ yields

$$
P_{\text {total }}=V_{c} I_{c}+V_{s} I_{s}=R_{\mathrm{m}}\left(I_{c}^{2}+I_{s}^{2}\right)+B_{m} \omega^{2}+J_{\mathrm{m}} \omega \frac{\mathrm{d} \omega}{\mathrm{d} t}
$$

Separating the equation into thermal and mechanical parts gives

$$
\begin{aligned}
P_{\text {therm }} & =R_{\mathrm{m}}\left(I_{c}^{2}+I_{s}^{2}\right)+B_{m} \omega^{2} \\
P_{\text {mech }} & =J_{\mathrm{m}} \omega \frac{\mathrm{d} \omega}{\mathrm{d} t}
\end{aligned}
$$

Even though the wheel contains large energy, mechanical power is used only when the speed is changing, which happens slowly. If the speed is decreasing, the spin motor can be used as a generator to supply power, now a negative quantity, back into the driving system. This self-powering mode is used during the braking mode of the ISS CMG. The thermal power $P_{\mathrm{th}}$ is always positive and represents the heat generating losses in the motor. This can be used to heat the motor structure and thus provide readings for a temperature sensor.

Because the state equations are originally derived in terms of the effective RMS values of the currents and voltages, the power equation constructed above represents the average power used in the motor. even though sinusoidal changes in power are being neglected, there is another effect caused by the sinusoidal nature of the electrical quantities. In a simple LR circuit model of the spin motor, the inductance causes a phase change of the winding current relative to the driving voltage. This phase difference between $I(t)$ and $V(t)$ results in less power being used in the motor. Consequently, the power should be modified by the power factor equation

$$
P_{\mathrm{ave}}=I_{\mathrm{rms}} V_{\mathrm{rms}} \cos \theta
$$

Any nonzero phase angle will reduce the computed power. From elementary circuit theory, the phase angle between driving voltage and current in an LR circuit is $\arctan \frac{\omega L}{R}$. Using the impedance triangle in the complex plane, an expression for $\cos \theta$ becomes

$$
\cos \theta=\frac{1}{\sqrt{1+\frac{\omega L}{R}}}
$$


This power-factor is best used when the motor is in a non-steady-state mode, either increasing or decreasing its speed. When in constant speed mode the power factor is close to unity and could be neglected. For the ISS CMG, it is found that the power-factor resulted in a more realistic power usage value when in spin-up mode.

\section{Spin Motor Controller}

An adaptive proportional voltage controller is presented which adjusts the PWM voltages depending on several control modes for speed, current, and torque. There are five modes of operation for a CMG wheel. Nominal mode for normal operating speeds or changing speeds within a specific range. The Spin-up mode for spinning up the wheel from rest. The Braking mode for spinning down from a nominal speed and the CMGs are powered from the back EMF as long as the wheel speed is above 1300 RPM. Finally, the wheel is in Coasting mode, if the wheel is spinning down without any power neither from an external power source (RPCMs) nor an internal power source (back EMF). The voltage controller is used for each mode of operation. The voltage controller updates CMG spin motor's acceleration based on the current rate, past and current commanded rates for a given time step $\mathrm{d} t$. The input commanded rates are generated by a steering control law generally implemented in the flight software. A simple fixed gain proportional control with a feed forward term is used to determine the acceleration. Also, the current commanding gimbal rate is filtered based on the history of commanded and filtered rates.

The two electrical equations (22a and 22b) for the motor windings contain two free parameters for control of the motor speed; the sine and cosine drive voltages $V_{s}$ and $V_{c}$. A voltage function is required that will change the voltages in order to satisfy the desired conditions of speed, current and torque. For the ISS CMGs, it is realized that the relatively weak motor driving the heavy wheel constitutes a slowly changing system and thus in certain modes of operation it is assumed to be in a pseudo-steady-state condition. The steady-state versions of the state equations (22) where $\frac{\mathrm{d} I}{\mathrm{~d} t}=\frac{\mathrm{d} \omega}{\mathrm{d} t} \approx 0$ are

$$
\begin{aligned}
V_{s} & =R I_{s}+K_{\mathrm{e}} \omega \\
V_{c} & =R I_{c}+K_{\mathrm{e}} \omega \\
I_{s}+I_{c} & =\frac{B_{\mathrm{m}} \omega}{K_{\mathrm{t}}}
\end{aligned}
$$

Combining the electrical equations (35a and 35b) and taking the differentials gives

$$
\left.\mathrm{d}\left(V_{s}+V_{c}\right)=R\left(\mathrm{~d} I_{c}+\mathrm{d} I_{s}\right)+2 K_{\mathrm{e}} \mathrm{d} \omega\right)
$$

\section{A. Nominal Mode}

The nominal mode is the most often used control mode where the ISS flight software commands the CMG to spin at one of 16 pre-defined speeds ranging from 6270 to 6930 RPM with 6600 RPM being the nominal speed. Using Hall effect sensors the control circuit determines the speed error and converts this error to control current for the PWM drive. A simple proportional controller could be constructed using speed error as the error signal like the following equation

$$
\Delta V=G_{p}\left(1-\frac{\omega}{\omega_{\mathrm{cmd}}}\right)
$$

This method results in the currents not correlating with the speed change duration as on the ISS CMG. A better method is to use a current limiting controller which acts similar to the real CMG's current control as follows

$$
\Delta V=G_{p}\left(I_{\text {lim }}-\left|I_{c, s}\right|, \text { with the sign of }\left(\omega-\omega_{\mathrm{cmd}}\right)\right)
$$

The $I_{\lim }$ value is the nominal spin motor maximum current of $\approx 1.63 \mathrm{~A}$. The absolute value of the sine or cosine current is used so that the speed error provides the sign change for spin-up or spin-down. Finally, the 
proportional gain $G_{p}$ is tuned so that the wheel spin-up or spin-down durations between the nominal set of speeds matches the real CMGs. These times are roughly $3 \mathrm{~min}$ for spin-down or $5 \mathrm{~min}$ for spin-up. This model uses different gains for up-and-down speed changes.

For all control modes, once the motor attains a speed within 0.5 RPM of the commanded speed, the control voltages are immediately set to the steady-state voltage appropriate for that speed. From the steadystate equations (35a-35c), the steady-state voltage $V_{s s}$ becomes

$$
V_{s s}=\left(\frac{R_{m} B_{\mathrm{m}}}{2 K_{\mathrm{t}}}+K_{\mathrm{e}}\right) \omega_{\mathrm{cmd}}
$$

\section{B. Spin-up Mode}

One of the basic duties of the spin motor controller is to spin-up the wheel from a dead stop to its operating speed of 6600 RPM in approximately 7 hours. Figure 6 shows a plot of current versus RPM for this spin-up mode.

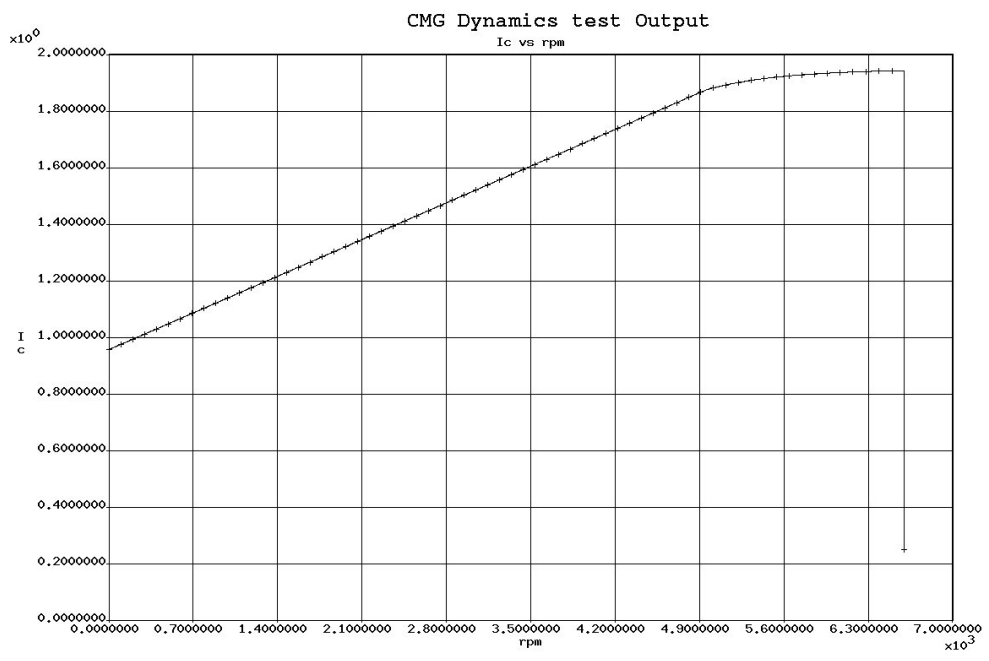

Figure 6: Spinup Current I vs RPM

\section{Linear Current Control}

The current follows a very linear trend from 0 to 5000 RPM and then gradually reaches a plateau of maximum current corresponding to the motor's torque limit at nominal speed. At this point it abruptly drops to steady-state values to overcome friction in the system and to maintain speed. The slope of the linear portion of the spin-up graph provides the desired current change per RPM $\left(\frac{\mathrm{d} I}{\mathrm{~d} \omega}\right)$ for the controller. The ISS CMGs exhibit a spin-up ramp of $0.000185 \frac{\mathrm{Amp}}{\mathrm{rpm}}=0.00177 \mathrm{Amp}-\mathrm{sec}$. Here only the slope of the current is of interest and not the actual values. Including the known current ramp value $\frac{\mathrm{d} I_{c}}{\mathrm{~d} \omega}$ in the differential relation for voltage from Eq. (36) gives

$$
\mathrm{d}\left(V_{s}+V_{c}\right)=R\left(\frac{\mathrm{d} I_{c}}{\mathrm{~d} \omega}+\frac{\mathrm{d} I_{s}}{\mathrm{~d} \omega}\right) \mathrm{d} \omega+2 K_{\mathrm{e}} \mathrm{d} \omega
$$

Assuming both sine and cosine drives operate identically allows to combine and simplify further to a single voltage control law for spin-up condition

$$
\Delta V=\left(R \frac{\Delta I}{\Delta \omega}+K_{\mathrm{e}}\right) \Delta \omega
$$


where $\frac{\Delta I}{\Delta \omega}=$ constant is the slope of the curve in Figure *. During spin-up, using Eq. 38, the driving voltages are incremented as $V_{c 1}=V_{c 0}+\Delta V$ and $V_{s 1}=V_{s 0}+\Delta V$. For starting from rest $(\omega=0)$, an initial voltage is needed to supply the starting torque; the smallest voltage step value for the CMG PWM power supply is 3.41 Volts.

\section{Maximum Torque Control}

The current plateau in the spin-up curve is modeled assuming the motor has reached some limiting maximum torque. Considering the original mechanical state equation Eq. 22c and substituting a torque value $\tau_{\max }$ for the motor currents term yields

$$
J_{\mathrm{m}} \frac{\mathrm{d} \omega}{\mathrm{d} t}=K_{\mathrm{t}}\left(I_{c}+I_{s}\right)-B_{\mathrm{m}} \omega=\tau_{\max }-B_{\mathrm{m}} \omega
$$

Solving for $\mathrm{d} \omega$ gives

$$
\mathrm{d} \omega=\frac{\left(\tau_{\max }-B_{\mathrm{m}} \omega\right)}{J_{\mathrm{m}}} \mathrm{d} t
$$

Combining all three steady-state equations (35a-(35c) that includes electrical and mechanical models gives rise to this single expression

$$
V_{c}+V_{s}=\left(\frac{R_{m} B_{\mathrm{m}}}{K_{\mathrm{t}}}+2 K_{\mathrm{e}}\right) \omega
$$

Once again assuming that both the drives operate equally $\left(V_{s}=V_{c}\right)$ and taking the differentials for Eq. (44) yields

$$
\mathrm{d} V=\left(\frac{R_{m} B_{\mathrm{m}}}{2 K_{\mathrm{t}}}+K_{\mathrm{e}}\right) \mathrm{d} \omega
$$

Now plugging in Eq. (43) into Eq. (45), the voltage differences become

$$
\Delta V=\frac{1}{J_{\mathrm{m}}}\left(\frac{R_{m} B_{\mathrm{m}}}{2 K_{\mathrm{t}}}+K_{\mathrm{e}}\right)\left(\tau_{\max }-B_{\mathrm{m}} \omega\right) \Delta t
$$

This control law is invoked during spin-up when the motor electrical torque exceeds the maximum value $\tau_{\max }$ such that the condition $K_{\mathrm{t}}\left(I_{c}+I_{s}\right)>\tau_{\max }$ is satisfied. The ISS CMG spin motor exhibits a $\tau_{\max }$ range of 37 to 39 oz.in. The 2 spin-up voltage control laws above will drive the wheel to 6600 RPM in seven hours which is the average of the true range of 6 to 8 hours.

\section{Braking Mode}

Another dynamic mode of spin-motor operation is the method used to spin-down the wheel to a lower speed. This mode, called active braking, inserts a number of large parallel resisters into the motor drive circuit to dissipate the back EMF current and slowly reduce wheel speed. There are two types of braking; normal and hard. They are distinguished by the amount of torque applied by limiting the motor current. Here, as in the spin-up mode, it can be viewed as another torque limiting control function which now decrements the driving voltages. Using the same maximum torque control function as before but with sign changes to slowly reduce driving voltages and reflecting the fact that now the system drag aids in slowing of the wheel, the change in voltage is expressed as

$$
\Delta V=-\frac{1}{J_{\mathrm{m}}}\left(\frac{R_{b} B_{\mathrm{m}}}{2 K_{\mathrm{t}}}+K_{\mathrm{e}}\right)\left(\tau_{\mathrm{b}}+B_{\mathrm{m}} \omega\right) \Delta t
$$

where $R_{b}$ contains the winding and braking resistances of approximately $80 \Omega$. And $\tau_{b}$ is either the normal braking torque of $15 \mathrm{oz}$.in or the hard braking value $37.5 \mathrm{oz}$.in.

When the wheel is in braking mode some of the back EMF voltage can be used to generate internal power to keep the power and PWM control circuits of the spin motor activated. This generator mode is 
required if external power is lost or a power supply has failed and the CMG can still be used to supply steering torque to the ISS down to a predetermined wheel speed. This mode continues until the wheel has slowed to 3600 RPM when the controller either continues with normal braking, or, if continued internal power is needed, switches to hard braking to supply more back EMF. In either case, when the wheel has reached 1300 RPM the motor torque ceases and is put into the coasting mode where the freewheeling rotor is slowed down by friction only.

\section{Coasting Mode}

The wheel enters the coasting mode if one of these two conditions are satisfied:

1. Spin motor is disconnected.

2. Spin motor is turned off and the wheel is not on internal power (wheel speed is $<1300$ RPM).

The coasting mode simply sets the sine and cosine drive voltages to zero so that the wheel follows the natural dynamics (Eq. 22c) for spinning down to a full stop.

\section{Simulation}

This section presents numerical simulations illustrating the behavior of a CMG spin motor's electromechanics, the voltage controller's performance for several modes of operation and how well the results matched with the real CMGs. Different wheel speed modes i.e, nominal, spin-up, braking and coasting are verified in this simulation. For each mode, how the wheel speed changes with time is shown and specifically for spin-up mode the current and power usage is illustrated. Table 1 provides the simulation parameters and their values.

Table 1: Spin Motor Design Parameters

\begin{tabular}{|c|c|c|}
\hline Parameter & Value & Units \\
\hline \hline Torque Constant, $K_{\mathrm{t}}$ & 0.08 & $\mathrm{Nm} / \mathrm{A}_{\text {peak }}$ \\
Electrical Constant, $K_{\mathrm{e}}$ & 0.014 & $V_{\text {peak }} / \mathrm{rpm}$ \\
Resistance (windings + on resistance), $R_{\mathrm{m}}$ & 3.56 & $\Omega /$ Phase \\
Inductance (coils), $L_{\mathrm{m}}$ & 0.002 & $\mathrm{H} / \mathrm{Phase}$ \\
Inertia, $J_{\mathrm{m}}$ & 7.1 & $\mathrm{~kg} \mathrm{~m}{ }^{2}$ \\
Drag Constant, $B_{\mathrm{m}}$ & 0.00005 & $\mathrm{Nm} / \mathrm{rad} / \mathrm{s}$ \\
\hline
\end{tabular}

The nominal speed mode tests the voltage controller's performance by increasing the speed from 6600 RPM to 6810 RPM. Figure 7 shows the speed increase which the controller achieved in $17 \mathrm{~min}$. The spin-up test is the most critical and sets up the conditions to speed up from rest (0 RPM) to 6600 RPM. As shown in Figure 8, the controller performed well spinning up to 6600 RPM in $7 \mathrm{hrs}$ (the real CMGs spin up in 6-8 hrs), which also implies that the RMS assumption is reasonable. The braking test turns off the spin motor power supply which sets the simulation mode to braking. Figure 9 shows that the spin-down to 1300 RPM happens in 7.8 hours during which CMGs are also powering the SM, IG and OG control cards using the back EMF. When the speed drops below 1300 RPM, the CMGs transition to coasting mode and turn off the power to the SM, IG and OG control cards which results in slower spinning down of the wheel. Figure 10 shows the results for the coasting mode only which is set by turning off the voltage supplies to the CMGs and disconnecting the spin motor. Coasting is a slow operation which can take up to 200 hours for the CMG to completely spin-down to rest.

The simulation results illustrate the interaction between the electrical system and the load dynamics and how these influence the overall performance of the system. The heat generated ends up in the thermal 


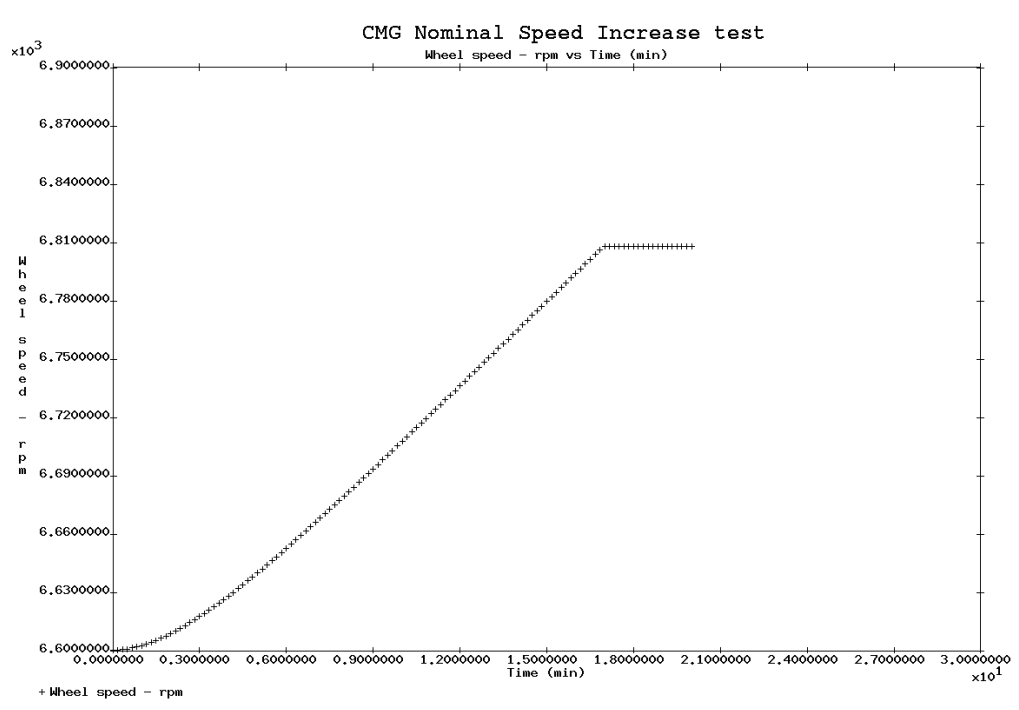

Figure 7: Nominal Speed Increase Test

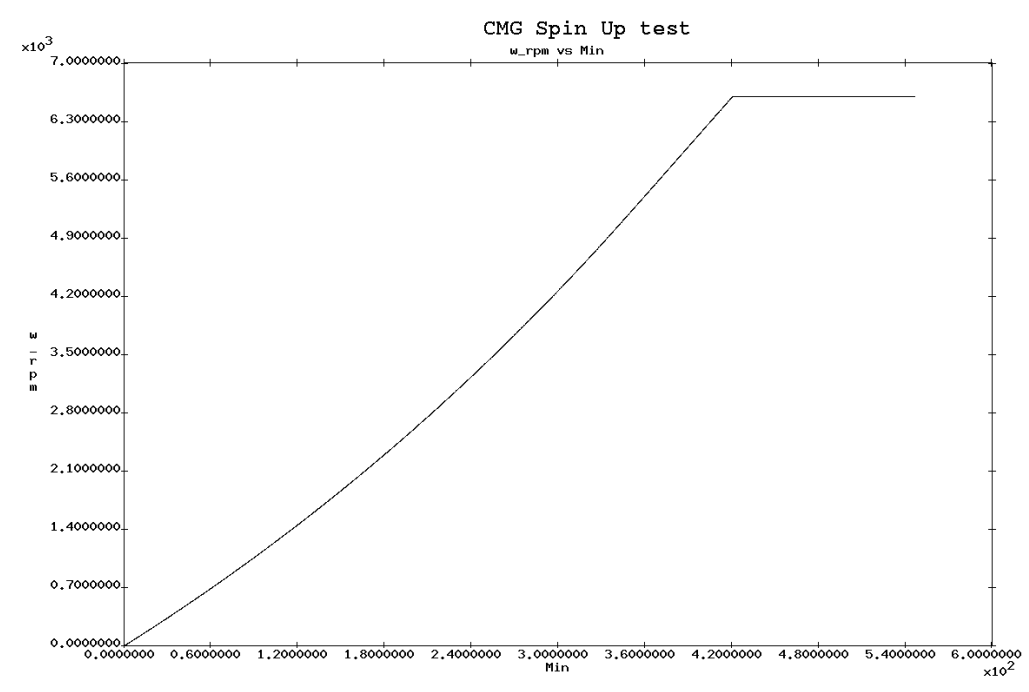

Figure 8: Spin-up Test

control subsystem (TCS) and the electrical power consumed goes to the electrical power subsystem (EPS) for effective (average) calculations of CMG power consumption. Essentially, the total power consumed in the motor is turned into a simple resistor and its value sent to the EPS system. The EPS solves for current and powers in the CMG as part of the solution for the whole ISS system. The CMG model development and testing is completed and is deployed as part of the TS21 simulation.

\section{Conclusion}

This paper presents simplified electromechanical motor and voltage controller models to simulate a PWM two-phase spin motor drive mechanism and its control cards. The basic principles of single-phase and two-phase brushless DC motors are discussed. And implicit Euler integrator is used to solve for the two phase currents, voltages, wheel speeds, torques and power with respect to time. Then the various spin motor voltage controllers are discussed for different wheel modes; nominal, spin-up, braking and coasting. The most interesting aspect of this work is that the results compare similarly to real CMG data which will aid the flight controllers with realistic training on the TS21 GNC-CMG simulation. The simple electromechanical and voltage controller models derived in this paper can be reused in other applications which use either a 


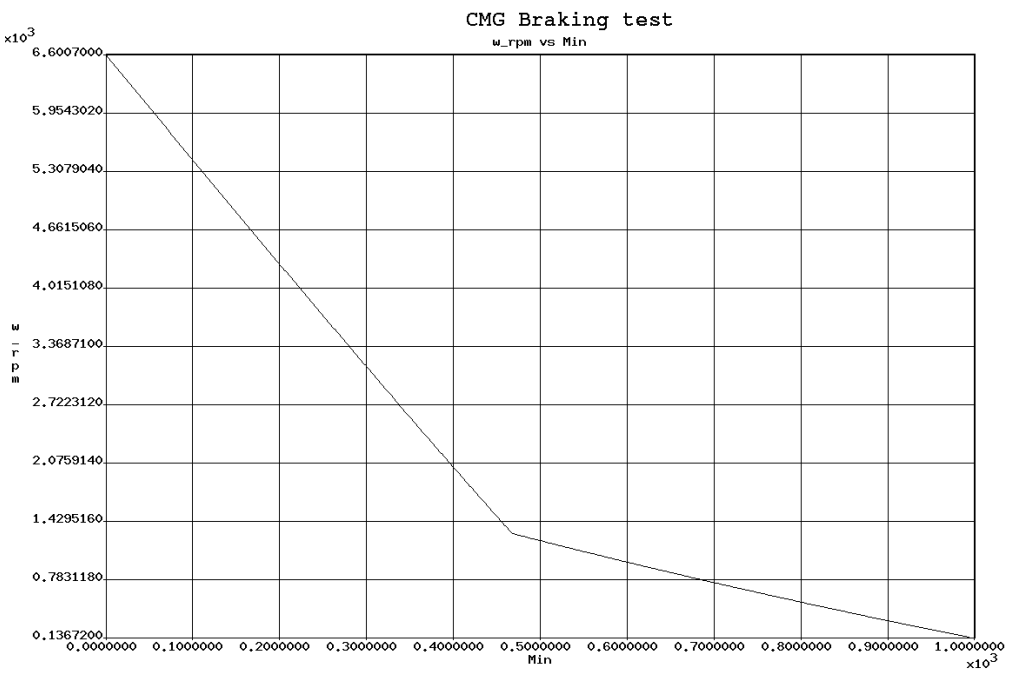

Figure 9: Braking Test

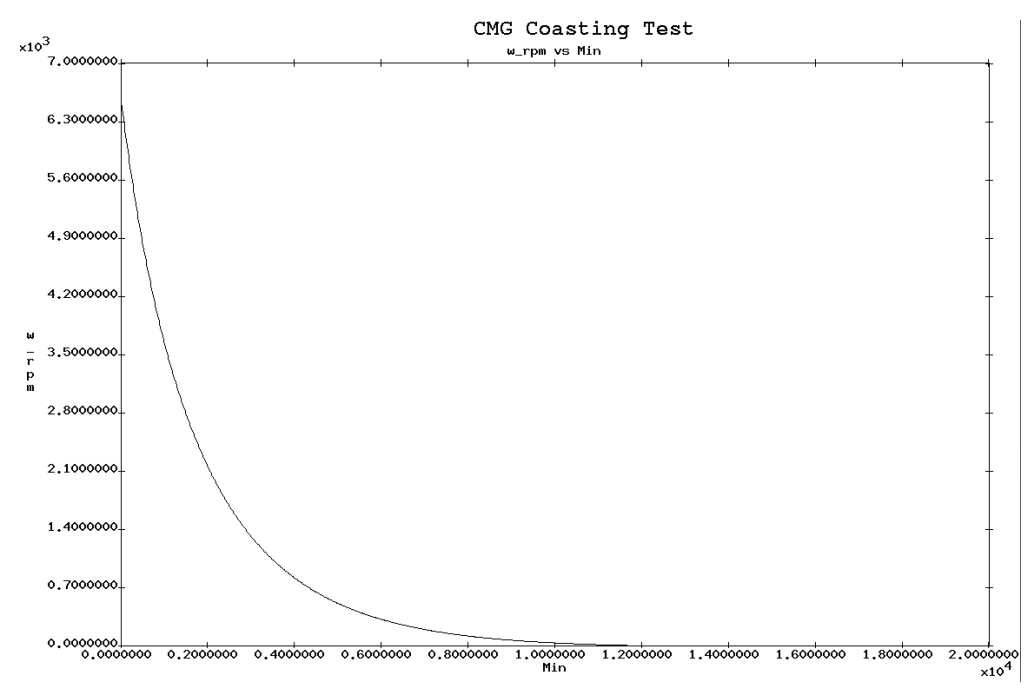

Figure 10: Coasting Test

single-phase or a two-phase DC motor assuming the current versus speed curves are known for the respective applications.

\section{Acknowledgments}

The authors of this paper would like to thank the Johnson Space Center engineering directorate under the Training Systems 21 contract. We also acknowledge the GNC flight controllers and trainers at JSC for providing valuable feedback.

\section{References}

${ }^{1}$ The Simulation and Graphics Branch, ER7, NASA, JSC, Houston, TX, http://er.jsc.nasa.gov/ER7/.

2"ADCO Console Handbook," International Space Station Attitude Determination and Control Officer (ADCO), Mission Operations Directorate Systems Division, JSC-36410, Johnson Space Center, NASA, Houston, TX, March 15, 2013.

${ }^{3}$ Stevenson, D., and Schaub, H., "Nonlinear Control Analysis of a Double-Gimbal Variable-Speed Control Moment Gyroscope," Journal of Guidance, Control, and Dynamics, Vol. 35, No. 3, May-June 2012.

${ }^{4}$ Schaub, H., and Junkins, J. L., Analytical Mechanics of Space Systems, 2nd ed., AIAA Education Series, AIAA, Reston, VA, Oct. 2009, pp. 178-188, 408-430. 
${ }^{5}$ Troung, V. L., Wolff, J. F., and Dravid, V. N., "Simulation of Flywheel Electrical System for Aerospace Applications," AIAA, Paper 2000-2908, 2000.

${ }^{6}$ Rodriguez D. F., "Electromechanical Motion Control Systems in Spacecraft Applications," AIAA, Paper 2006-7291, 2006, San Jose, California. 